\title{
Evaluación de la Cassia fistula como coagulante natural en el tratamiento primario de aguas residuales
}

\author{
Assessment of natural coagulant Cassia fistula in the primary \\ treatment of wastewater
}

\section{Avaliação da fístula Cassia como coagulante natural no tratamento primário das águas residuais}

\author{
Arnulfo A. Tarón-Dunoyer ${ }^{1}$, Luis E. Guzmán-Carrillo² ${ }^{2}$ Israel Barros-Portnoy ${ }^{3}$
}

1 Químico Farmacéutico, MSc, (C)PhD, Docente, Programa Ingeniería de Alimentos, Universidad de Cartagena, Facultad de Ingeniería, Campus Piedra de Bolívar. Cartagena-Bolívar, Colombia.

2 Ing. Alimentos, Esp, Docente, Programa de Ingeniería de Alimentos, Universidad de Cartagena, Facultad de Ingeniería, Campus Piedra de Bolívar. Cartagena-Bolívar, Colombia.

3 Químico Farmacéutico, MSc, Ciencia y Tecnología de Alimentos. Docente Universidad del Atlántico, Facultad de Química y Farmacia, Km 7 Antigua vía Puerto Colombia, Barranquilla, Colombia.

Email: atarond@unicartagena.edu.co

Recibido: 11 de mayo de 2015

Aceptado: 27 de junio de 2017

\begin{abstract}
Resumen
Diferentes compuestos naturales extraídos de plantas se han usado para el tratamiento de aguas residuales por muchos siglos. Estos en su gran mayoría derivan de semillas, hojas, cortezas o savia, raíces y frutos de árboles y plantas. En este trabajo se evaluó la utilización del polvo de la semilla de la Cassia fístula como coagulante natural en el tratamiento primario de aguas residuales domésticas, estableciendo su dosis óptima mediante la prueba de jarras y determinando los parámetros fisicoquímicos de DBO5, DQO, conductividad, color, turbidez, alcalinidad total, y dureza total. Se utilizó como muestra de estudio agua residual doméstica tomada de una estación de bombeo de la ciudad de Cartagena de Indias (Colombia). Se encontró una dosis óptima del coagulante natural comprendida entre 15-25 mg/L, obteniendo valores finales de 30.25 NTU en la turbidez y 84 UC de color respectivamente. Los valores de $\mathrm{pH}$ y alcalinidad total no presentaron mayores variaciones. Los valores de los parámetros turbidez y color, al igual que el $\mathrm{pH}$, alcalinidad total y dureza total se corresponden con los reportados por diferentes autores para este tipo de agua, lo cual demuestra que la C. fistula es un coagulante natural, prometedor y eficaz para la sustitución de coagulantes inorgánicos en el proceso de coagulación de aguas residuales
\end{abstract}

Palabras clave: dosis óptima, estabilidad, floculación.

\begin{abstract}
Different natural compounds extracted from plants have been used for the treatment of wastewater for many centuries. These mostly derived from seeds, leaves, bark or sap, roots and fruits of trees and plants. In this paper the use of seed powder Cassia fistula as a natural coagulant was studied. Establishing optimum dose by jar testing and determining the parame-
\end{abstract}


ters of DBO5, DQO, conductivity, color, turbidity, total alkalinity and total hardness; using wastewater pumping station of the city of Cartagena de Indias (Colombia). Natural optimal coagulant dose between 15-25 mg/L is found. Obtaining final values of 30.25 NTU turbidity and 84 color UC's respectively. The values of $\mathrm{pH}$ and total alkalinity no major variations. The values of the turbidity and color parameters like $\mathrm{pH}$, total alkalinity and total hardness corresponding with those reported by different authors, which shows that $C$. fistula is a natural, promising and effective coagulant for the substitution of inorganic coagulants in the process of coagulation of wastewater

Key words: flocculation, optimal dosage, stability.

\section{Resumo}

Diferentes compostos naturais extraídos de plantas foram utilizados para o tratamento de águas residuais durante muitos séculos. Estes em sua grande maioria derivam de sementes, folhas, cascas ou seiva, raízes e frutos de árvores e plantas. Neste documento, a utilização de pó de sementes de Cassia fístula como um coagulante natural no tratamento primário de águas residuais domésticas foi avaliada estabelecendo a dose óptima mediante o teste de frasco determinando os parâmetros físico-químicos de CBO5, DQO, condutividade, cor, turbidez, alcalinidade total e dureza total. Foi utilizada como amostra de estudo água residual doméstica retirada de uma estação de bombeamento na cidade de Cartagena de Índias (Colômbia. Foi encontrada uma dose ótima do coagulante natural compreendido entre 15-25 mg / L, obtendo valores finais de 30,25 NTU na turbidez e 84 UC de cor, respectivamente. $\mathrm{O}$ pH e a alcalinidade total não mostraram maiores variações. Os valores da turbidez e a cor, como o pH, alcalinidade total e dureza total de parâmetros correspondem aos relatados por outros autores para a água, o que mostra que a C. fístula é um coagulante natural, promissora e eficiente para a substituição de coagulantes inorgânicos no processo de floculação de águas residuais

Palavras-chave: dose ideal, estabilidade, floculação

\section{Introducción}

En el tratamiento de agua turbia superficial y de aguas residuales domésticas e industriales la coagulación es un proceso esencial, siendo uno de los pasos principales para la remoción de turbidez, color y materia orgánica, que se logra generalmente utilizando coagulantes (Katayon et al., 2007). Muchos de ellos como el cloruro férrico, sulfato de aluminio, cloruro de polialuminio y carbonato de calcio, son utilizados para eliminar las impurezas y las partículas coloidales de las aguas residuales y naturales (Bergamasco et al., 2009). Sin embargo, existen desventajas asociadas al uso de estos coagulantes, como altos costos de adquisición, producción de grandes volúmenes de lodos y el hecho de que afectan significativamente el $\mathrm{pH}$ del agua tratada (Yin, 2010).

Los extractos de plantas naturales tales como Moringa oleífera, Jatropha curcas, Cyamopsis tetragonoloba, Strychnos potatorum, Hibiscus sabdariffa y Clidemia angustifolia se han usado para la purificación del agua por muchos siglos. La mayoría de estos coagulantes se derivan de semillas, hojas, cortezas o savia, raíces y frutos de árboles y plantas o pueden extraerse de microorganismos, animales o tejidos vegetales (Yuan and Manh, 2015; Pritchard et al., 2009); estos coagulantes muestran ser biodegradables y se presume que son seguros para la salud humana (Šćiban et al., 2009). Además, los coagulantes naturales producen lodos menos voluminosos en cantidad y rápidamente biodegradables que su contraparte el alumbre (Bhuptawat et al., 2007). El uso de materiales naturales de origen vegetal para clarificar las aguas crudas turbias no es una idea nueva. Los coagulantes naturales se han usado tradicionalmente en el tratamiento de aguas residuales en diferentes áreas e incluso en áreas tropicales (Guzmán et al., 2013). En particular, la Moringa oleifera originaria de Sudán se ha clasificado como uno de los mejores extractos de plantas para el tratamiento del agua (Pritchard et al., 2010). Este árbol es considerado mundialmente como el árbol milagroso, debido a que cada parte del árbol de Moringa puede usarse en la alimentación, la medicación y para propósitos industriales (Khalafalla et al., 2010).

La caña fístula, carao o cañadonga es un árbol natural de América Central y las zonas costeras de las Antillas, perteneciente a la familia Fabaceae género Cassia. En Colombia, México y probablemente en otros países, también se le conoce como lluvia de oro (Cassia fistula Golden-Shower). Es un árbol pequeño de hasta $5 \mathrm{~m}$ de altura por $30 \mathrm{~cm}$ de diámetro, con la corteza gris verdoso, hojas compuestas, pinnadas, con 4 a 8 pares de hojuelas. Las flores son de color amarillo intenso y están dispuestas en grandes racimos colgantes. Los frutos son vainas alargadas, delgadas, cilíndricas, negras, de aproximadamente $50 \mathrm{~cm}$ de largo. El nombre del género Cassia proviene de los antiguos griegos, quienes lo aplicaban a una serie de plantas con propiedades terapéuticas. El epíteto fístula proviene del latín que significa "tubo", en alusión a la forma de los frutos (Sánchez y Cascante, 2008). 
Ante este panorama; y conociendo que la utilización de materiales de origen vegetal como el polvo de semillas de árboles y materiales comestibles entre otros, han sido mencionados y utilizados como coagulantes naturales para la clarificación de aguas; se plantea la posibilidad de sustituir los agentes coagulantes de uso común por el polvo de semillas de C. fistula como agente coagulante natural y su aplicabilidad en el tratamiento primario de aguas residuales domésticas (Bui et al., 2016)

\section{Materiales y métodos}

Para el desarrollo de esta investigación se utilizó agua residual tomada de una estación de bombeo de la ciudad de Cartagena de Indias - Colombia. La recolección de la muestra se realizó a las nueve de la mañana hora en que se supone que se alcanza el pico máximo de descargas que pasa por esta estación de bombeo y en el que el agua se caracteriza por presentar una elevada turbidez.

El coagulante natural se obtuvo conforme al esquema propuesto por Yin (2010). Las semillas de C. fistula se recolectaron de forma manual, desechando aquellas que presentaron daños por insectos. Una vez recolectadas y seleccionadas, estas se expusieron al sol durante un periodo aproximado de 8 días. Las semillas secas, se molieron en un molino mecánico helicoidal Marca Pulvex Modelo 95, posteriormente se pasaron por un tamiz con numero de malla 10. Esta operación se repitió varias veces hasta obtener un polvo de consistencia fina o agente coagulante. La solución del coagulante se preparó disolviendo 25 g de polvo de semilla en $100 \mathrm{~mL}$ de agua destilada

\section{Prueba de coagulación}

Para determinar las propiedades coagulantes del extracto acuoso de semillas de C. fistula se utilizó la prueba de jarras estándar descrita otros por Satterfield (2005). El procedimiento consistió en tomar siete vasos de precipitados en los cuales se vertieron $500 \mathrm{~mL}$ de agua residual obtenida de la estación de bombeo, utilizando uno como control; los otros seis se dosificaron con los extractos de semilla de C. fistula con concentraciones de 120, 140, 150, 160, 180 y 200 mg/L respectivamente. El agua residual y la mezclada con el agente coagulante fueron sometidas inicialmente a una agitación rápida a $100 \mathrm{rpm}$ durante 1 minuto, seguida de agitación lenta a $40 \mathrm{rpm}$ durante $30 \mathrm{mi}$ nutos; por último, se permitió un tiempo de sedimentación de 60 minutos. Finalmente se tomó con ayuda de una pipeta graduada $20 \mathrm{~mL}$ del agua clarificada sobrenadante para su análisis. Los ensayos se realizaron a temperatura ambiente y el equipo utilizado fue el Floculador digital E\&Q F6-300.

Los parámetros fisicoquímicos de color, turbidez, $\mathrm{pH}$, alcalinidad, conductividad, DBO5, DQO y dureza se evaluaron por triplicado en el agua residual y en el agua tratada con el agente coagulante utilizando la metodología propuesta por la APHA y col. (2012). El color real y aparente se midió por comparación visual de la muestra utilizando un colorímetro Lovibond PFX 195 aplicando el método 2120B y los resultados de la evaluación de color se expresaron en unidades de platino-cobalto (UPC). La turbidez se determinó por el método nefelométrico (método 2130B); el cual mide la turbidez en unidades de turbidez nefelométricas (UTN) utilizando como solución estándar un polímero de formalina. El equipo utilizado fue el turbidimetro Turbiquant 3000 IR. Para la medición del potencial de hidrogeno se utilizó un potenciometro digital (Bench $\mathrm{pH} /$ Conductivity meter PC 510), expresando los resultados en unidades de $\mathrm{pH}$ (método $4500-\mathrm{H}^{+} \mathrm{B}$ ). La alcalinidad se determinó por titulación y es expresada en mg de $\mathrm{CaCO}_{3} / \mathrm{L}$ (método 2320 B). La dureza se realizó por titulación utilizando como agente titulante una solución EDTA y es expresada en mg de $\mathrm{CaCO}_{3} / \mathrm{L}$ (Método 2340 C). Para la determinación de la DQO y DBO5 se utilizó el método propuesto por los métodos estándares para el análisis de aguas y aguas residuales de la APHA (2012). Estos parámetros solo fueron determinados para la dosis de $160 \mathrm{mg} / \mathrm{L}$ del agente coagulante.

\section{Resultados y discusión}

Los valores correspondientes a la calidad del agua residual domiciliaria se presentan en la Tabla 1. En ella se puede observar que el valor de la $\mathrm{DBO}_{5}$ es el $63 \%$ del valor obtenido por la DQO, mientras que los valores obtenidos para la turbidez y el color aparente fueron de 95 NTU y 280 UPC respectivamente.

Tabla 1. Parámetros de calidad del agua residual.

\begin{tabular}{|l|c|c|}
\hline \multicolumn{1}{|c|}{ Parámetro } & Valor & Unidad \\
\hline Turbidez & $95.00 \pm 1.00$ & $\mathrm{NTU}$ \\
\hline Color aparente & $280.0 \pm 0.60$ & $\mathrm{UPC}$ \\
\hline Alcalinidad Total & $258.0 \pm 1.00$ & $\mathrm{mg} \mathrm{CaCO} / \mathrm{L}$ \\
\hline Dureza Total & $510.0 \pm 0.57$ & $\mathrm{mg} \mathrm{CaCO} 3 / \mathrm{L}$ \\
\hline DBO5 & $152.0 \pm 0.81$ & $\mathrm{mg} / \mathrm{L}$ \\
\hline DQO & $241.5 \pm 0.76$ & $\mathrm{Mg} / \mathrm{L}$ \\
\hline Conductividad & $1156.9 \pm 0.80$ & $\mu \mathrm{Scm}^{-1}$ \\
\hline $\mathrm{pH}$ & $7.400 \pm 0.01$ & \\
\hline
\end{tabular}


En la tabla 2 se aprecian los resultados obtenidos del efecto del coagulante en el color y que permiten afirmar que una dosis de $160 \mathrm{mg} / \mathrm{L}$ del coagulante reduce en un $70 \%$ la demanda bioquímica de oxigeno (DBO5), pero en sentido contrario no se evidencian cambios importantes en la demanda química de oxigeno (DQO) debido posiblemente a la presencia de compuestos orgánicos no biodegradables por métodos convencionales y refractarios a la oxidación biológica; estos valores demuestran lo importante y eficiente que resulta utilizar este coagulante para disminuir la carga orgánica presente en un cuerpo de agua residual.

En la figura 1 se observa la variación del color residual. A medida que aumenta la dosis de coagulante, el color disminuye alcanzando un valor mínimo de 84 UPC para una dosis de coagulante de $160 \mathrm{mg} / \mathrm{L}$. El resulta-

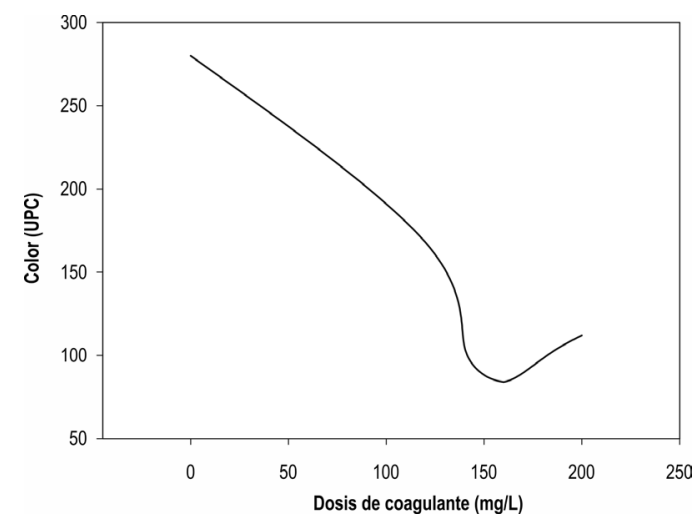

Figura 1. Comportamiento del Color en relación a la dosis de Polvo de Semilla de Cassia fistula.

do obtenido en esta investigación demuestra que la dosis de coagulante de $160 \mathrm{mg} / \mathrm{L}$ reduce los valores de color hasta 84 unidades, siendo la remoción de color del $70 \%$ con respecto a su valor inicial.

La figura 2 muestra que el aumento progresivo de las dosis del coagulante a base de polvo de semilla de la C. fístula 120, 140, 160, 180 y 200 mg/L condujo a una disminución de la turbidez. Con una dosis de 160 ppm de coagulante se alcanzó un valor mínimo para la turbidez de 30.25 UTN, para una remoción de $68,15 \%$ respecto de su valor inicial, estos resultados se corresponden con los encontrados por autores como Prakashy col (2014), pero utilizando como coagulante semillas de Moringa oleífera.

Para las dosis de 180 y $200 \mathrm{mg} / \mathrm{L}$ los porcentajes de remoción disminuyeron hasta un $50 \%$, este comportamiento puede estar muy relacionado con la reestabilización de

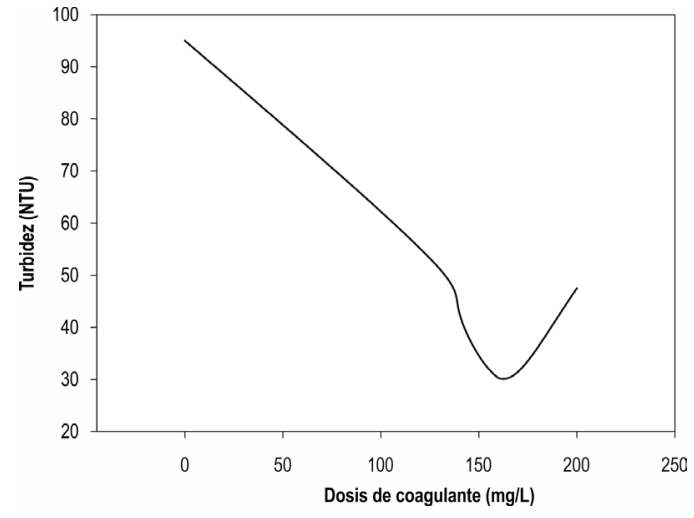

Figura 2. Remosión de Turbidez (\%) Vs dosis de coagulante.

las cargas presentes y que la actividad de la coagulación depende favorablemente de la relación óptima entre la dosis de coagulante y el catión bivalente en el agua. (Okuda et al., 2001). Los mecanismos postulados por los cuales los polielectrolitos presentes en la C. fistula pueden provocar la floculación son la neutralización de cargas y, la union y/o parche electrostático. (Petzold et al., 2003; Buchhammer et al., 2003; Schward, 2004)

Generalmente se cree que los polimeros con peso molecular bajo tienden a adsorber y/o neutralizar las cargas opuestas de las particulas, mientras que los polimeros de cadena larga y altos pesos moleculares pueden llevar a la floculación; en tales casos, los segmentos de una sola cadena polimerica se atan a mas de una particula.

El uso de polielectrolitos en lugar de los coagulantes inorganicos permite utilizar dosis mas bajas de coagulante, genera un menor volumen de lodo, la variación de la carga iónica del agua tratata es menor y hay una reducción de los costos en el orden del 30\%.

En la figura 3 se muestran las variaciones del $\mathrm{pH}$ para las diferentes dosis de coagulante. Con relación a la dosis del coagulante aplicado, el pH presentó una tendencia general a mantenerse con el incremento de la dosis del coagulante. En términos generales se puede

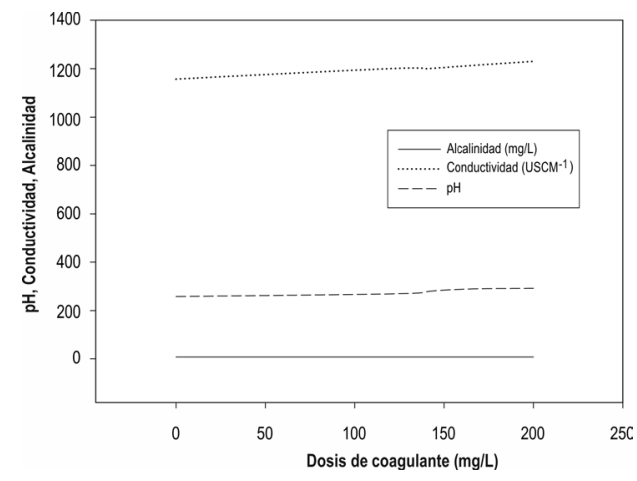

Figura 3. Comportamiento del $\mathrm{pH}$, alcalinidad y Conductividad en relación a la dosis de coagulante. 
afirmar que un aumento en la concentración de 120 a $180 \mathrm{mg} / \mathrm{L}$ no provocó cambios relevantes en el pH del medio (Shaw and Wu, 2015; Shaw and Wu, 2014; Bolto Gregory, 2007).

La alcalinidad total y la dureza total no presentaron variación con respecto a la dosis de coagulante aplicado. Con una dosis de $160 \mathrm{mg} / \mathrm{L}$ de coagulante, la dureza presentó una disminución de $20 \%$ aproximadamente con respecto a su valor inicial. A partir de una concentración inicial de $747 \mathrm{mg} / \mathrm{L}$ de sólidos totales en el agua residual, esta va disminuyendo a medida que se eleva la concentración de coagulante. A una dosis de $120 \mathrm{mg} / \mathrm{L}$ los sólidos totales disminuyen en un $60 \%$ de su valor inicial, llegando a un $74 \%$ cuando la dosis es de $160 \mathrm{mg} / \mathrm{L}$ y posteriormente se eleva la concentración de sólidos cuando se emplean dosis de 180 y 200 mg/L de coagulante. Este aumento en la concentración de sólidos al aumentar la dosis de coagulante radica en la restauración del sistema por la disolución de los flóculos formados (Guzmán et al., 2015)

En la Tabla 2 se muestran los resultados de esta investigación, los cuales evidencian la eficiencia del extracto acuoso del polvo de semilla de la C. fístula como coagulante primario sin la adición de sustancias químicas tales como óxido de calcio, hidróxido de calcio, hidróxido de sodio o carbonato de sodio, las cuales son requeridas cuando se usa el sulfato de aluminio para evitar el incremento de la acidez del agua, que la hace peligrosamente corrosiva.

Utilizando dosis de $160 \mathrm{mg} / \mathrm{L}$ de extracto acuoso del polvo de semilla de C. fistula se logran disminuciones del $68.2 \%$ para turbidez, $70 \%$ en color, $20 \%$ en dureza total, $70 \%$ en la DBO5 y $0.83 \%$ para la DQO, con base en estos resultados se puede inferir que se da una coagulación primaria adecuada. El extracto acuoso del polvo de semilla de la C. fistula no afectó significativamente los valores de $\mathrm{pH}$ para la dosis óptima de $160 \mathrm{mg} / \mathrm{L}$ del coagulante, por encontrarse estos dentro de los rangos establecidos en la Resolución 2115 de 2007.

En diferentes escritos se sugiere que las proteínas presentes en los materiales vegetales utilizados, son los ingredientes activos responsables de la coagulación. Al comparar el contenido de proteína del polvo de semilla de la C. fistula con los contenidos de proteína de los materiales vegetales: Castaño (Aesculushyppocastanum), Roble común (Quercusrobur), Roble turco (Quercuscerris) y Castaño europeo (Castanea sativa) utilizados por Šciban (2009), se encuentra que son similares en sus contenidos de proteínas.

\section{Conclusiones}

El presente estudio propone el uso del polvo de la semilla de la $C$. fistula como un coagulante natural alternativo para el tratamiento primario de aguas residuales, el test de jarras confirma su poder coagulante encontrando que con una dosis de $160 \mathrm{mg} / \mathrm{L}$ de agente coagulante, se alcanzan valores mínimos para el color de 84 UPC y para la turbidez de 30.25 NTU. El polvo de semilla de la C. fistula no afectó los valores de $\mathrm{pH}$, pero si los de alcalinidad total y dureza total para una dosis óptima de $160 \mathrm{mg} / \mathrm{L}$ de coagulante, por encontrarse estos resultados en los rangos permitidos para aguas residuales no requiere de la adición de sustancias químicas para ajustar estos valores según lo establecido por la norma colombiana vigente para descarga de aguas residuales.

Tabla 2. Resumen de los resultados obtenidos para las diferentes dosis de coagulante

\begin{tabular}{|c|c|c|c|c|c|c|c|c|}
\hline \multirow{2}{*}{ Parámetros } & \multirow{2}{*}{$\begin{array}{l}\text { Agua } \\
\text { residual }\end{array}$} & \multirow{2}{*}{ Unidades } & \multicolumn{6}{|c|}{ Dosis de coagulante $(\mathrm{mg} / \mathrm{L})$} \\
\hline & & & 120 & 140 & 160 & 180 & 200 & $\mathrm{Al}_{2}\left(\mathrm{SO}_{4}\right)_{3}$ \\
\hline Conductividad eléctrica & 1156.9 & $\mu \mathrm{Scm}^{-1}$ & 1200 & 1200 & 1210 & 1220 & 1230 & 1330 \\
\hline Turbidez & 95.00 & NTU & 55.10 & 42.75 & 30.25 & 36.10 & 47.50 & 9.500 \\
\hline Color real & 280.0 & UPC & 168.0 & 105.0 & 84.00 & 98.00 & 112.0 & 42.00 \\
\hline Dureza total & 510.0 & $\mathrm{mg} \mathrm{CaCo3} / \mathrm{L}$ & 459.0 & 453.9 & 408.0 & 411.9 & 438.6 & 331.5 \\
\hline $\mathrm{DBO}_{5}$ & 152.0 & $\mathrm{mg} / \mathrm{L}$ & & & 46.20 & & & 31.92 \\
\hline DQO & 241.5 & $\mathrm{mg} / \mathrm{L}$ & & & 239.5 & & & 245.0 \\
\hline Sólidos totales & 747.0 & $\mathrm{mg} / \mathrm{L}$ & 298.8 & 261.4 & 194.2 & 209.1 & 224.1 & 149.4 \\
\hline Alcalinidad & 258.0 & $\mathrm{mg} \mathrm{CaCo3/L}$ & 269.0 & 278.0 & 288.0 & 291.0 & 292.0 & 290.0 \\
\hline $\mathrm{Ph}$ & 7.400 & & 7.480 & 7.490 & 7.480 & 7.460 & 7.460 & 7.300 \\
\hline
\end{tabular}




\section{Referencias}

APHA (2012). Sthandar Methods for the Water and Wasterwater, 19 Ed. Washington D.C.

Bergamasco R, Bouchard C, Vieira da Silva F, Hespanhold M, Reis $M$, Fagundes MR. An application of chitosan as a coagulant/ flocculant in a microfiltration process of natural water. Desalination. 2009;245:205-213.

Bhuptawat H, Folkard GK, Chaudhari S. Innovative physico-Chemical treatment of wastewater incorporating Moringaoleifera seed coagulant. Journal of Hazardous Materials. 2007;142:477-482.

Bolto B, Gregory J. Organic polyelectrolytes in water treatment. Wat. Res. 2007;41:2301-2324

Buchhammer HM, Mende M, Oelmann M. Formation of monosized polyelectrolyte complex dispersions: effects of polymer structure, concentration and mixing conditions. Colloids and Surfaces A: Physicochem. Eng. Aspects 2003;218:151-159.

Bui HM, Perng YS, Duong HGT. The use of artificial neural network for modeling coagulation of reactive dye wastewater using Cassia fistula Linn(CF) gum. Journal of Environmental Science and Management. 2016;19(1):1-8.

Colombia. Ministerio de la Protección Social. Resolución 2115 de junio de 2007. Por medio de la cual se señalan características, instrumentos

Guzmán L, Tarón A, Nuñez A. Polvo de la semilla Cassia fistula como coagulante natural en el tratamiento de agua cruda Biotecnología en el Sector Agropecuario y Agroindustrial. 2015;13(2):123-129.

Guzmán L, Villabona A, Tejada, C. Reducción de la turbidez del agua usando coagulantes naturales: Una revisión. Actualidad \& Divulgación Científica. 2013;16(1):253-262.

Katayon S, Noor M, Kien Tat W, Halim GA, Thamer AM, Badronisa Y. Effect of natural coagulant application on microfiltration performance in treatment of secondary oxidation pond effluent. Desalination. 2007;204:204-212.

Khalafalla M, Abdellatef E, Dafalla H, Nassrallah A, Aboul-Enein $\mathrm{K}$, Lightfoot D, El-Deeb F, El-Shemy H. Active principle from $\mathrm{Mo}^{-}$ ringa oleifera Lam Leaves effective against two leukemias and a hepatocarcinoma. Afr. J. Biotechnol. 2010;9(49):8467-8471.

Okuda T, Baes A, Nishijima W, Okada M. Coagulation mechanism of salt solution extracted active component in Moringa oleifera seeds. Wat. Res. 2001;35(3):830-834.

Petzold G, Mende M, Lunkwitz K, Schwarz S, Buchhammer HM. Higher efficiency in the flocculation of clay suspensions by using combinations of oppositely charged polyelectrolytes., Colloids and Surfaces A: Physicochem. Eng Aspects. 2003;218:47-57.

Prakash NB, Sockan V, Jayakaran P. Waste Water Treatment by Coagulation and Flocculation. International Journal of Engineering Science and Innovative Technology (IJESIT). 2014;3(2):479-484.

Pritchard M, Craven T, Mkandawire T, Edmonson AS, O'neill JG. Comparison between Moringa oleifera and chemical coagulant in the purification of drinking water - An alternative sustainable solution for developing countries. Physics and chemistry of the Earth. 2010;35:798-805.
Pritchard M, Mkandawire T, Edmondson A, O'neill J, Kululanga G. Potential of using plant extracts for purification of shallow well water in Malawi. Physics and Chemistry of the Earth, Parts A/B/C., 2009;34(13-16):799-805.

Sánchez J, Cascante A. 2008. Árboles ornamentales del Valle Central de Costa Rica: especies con floración llamativa. San José (Costa Rica): $1^{\underline{a}}$ ed. Instituto Nacional de Biodiversidad (INBio), Museo Nacional. Pp.30

Satterfield Z. Tech Brief. Jartesting. Spring. 2005;5(1):1-4

Šćiban M, Klašnja M, Antov M, Škrbiš B. Removal of water turbidity by natural coagulants obtained from chestnut. Biores Techn. 2009;100(24):6639-6643.

Shak KPY, Wu TY. Optimized use of alum together with unmodified Cassia obtusifolia seed gum as a coagulant aid in treatment of palm oil mill effluent under natural $\mathrm{pH}$ of wastewater. Industrial Crops and Products. 2015;76:1169-1178.

Shak KPY, Wu TY. Coagulation-flocculation treatment of high-strength agro-industrial wastewater using natural Cassia obtusifolia seed gum: treatment efficiencies and flocs characterization. Chemical Engineering Journal. 2014;256:293-305.

United States. 2012. The American Water Works Association (AWWA). Standard Methods for the Examination of Water and Wastewater. New York (United States): ed. 0022. Pp.1496.

Yin C. Emerging usage of plant-based coagulants for water and wastewater treatment. Process Biochem. 2010;45:1437-144.

Yuan SP, Manh HB. The feasibility of Cassia fistula gum with polyaluminum chloride for the decolorization of reactive dyeing wastewater. Journal of Serbian Chemical Society. 2015;80(1):115-125.

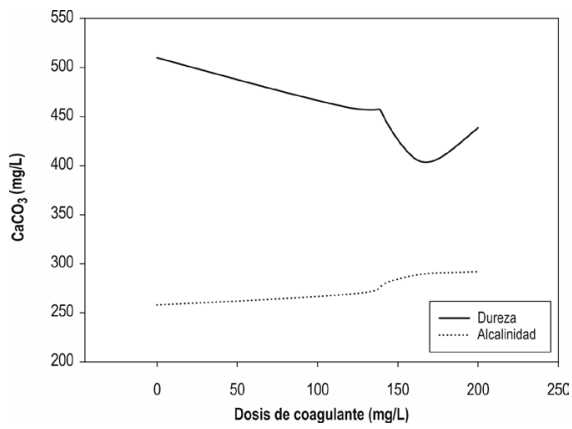

Figura 4. Comportamiento de la dureza y alcalinidad con respecto a la dosis de coagulante.

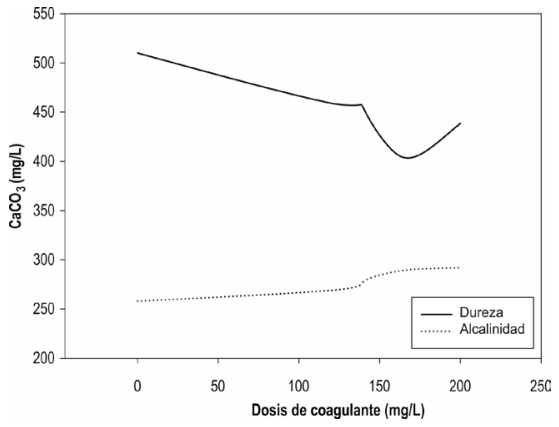

Figura 5. Sólidos totales con respecto a la dosis de coagulante. 